\title{
The effect of chronic prenatal hypoxia on the development of mature neurons in the cerebellum
}

Keumyoung So ${ }^{1}$, Yoonyoung Chung ${ }^{2}$, Hyunyoung Lee ${ }^{1}$, Eunyoung Kim ${ }^{3}$ and Yonghyun Jeon ${ }^{2 *}$

\begin{abstract}
Background: Adverse intrauterine circumstances can result in abnormal brain development, and can contribute to many neurological disorders such as cerebral palsy and cognitive and behavioral deficits. These neurological problems are caused by conditions that cause chronic placental insufficiency (CPI), such as hypoxia and acidemia. Hypoxia has been implicated in structural alterations of the cerebellum during development; however, the changes to the cerebellar external granular layer (EGL) induced by chronic prenatal hypoxia are not well understood. We therefore investigated the effect of chronic prenatal hypoxia on the development of mature neurons in the EGL using the guinea pig CPI model.

Methods: Unilateral uterine artery ligation was performed at 30 to 32 days of gestation (dg) - with term defined as approximately $67 \mathrm{dg}$. At $50 \mathrm{dg}, 60 \mathrm{dg}$, and one week after birth, fetuses and newborns were sacrificed and assigned to either the growth-restricted (GR) or control (no ligation) group. After fixation, dissection, and sectioning of cerebellar tissue from these animals, immunohistochemistry was performed with antibodies raised to hypoxia-induced factor 1a (Hif1a), Pax6, NeuroD, and NeuN.
\end{abstract}

Results: The induction of hypoxia was confirmed by the presence of Hif1 a immunoreactivity in the EGL of the GR (but not control) fetuses. The only other cellular immunoreactivity found in any of the tissues was to the NeuN antibody, which is a marker of mature neurons. The proportion of NeuN-immunoreactive (NeuN-IR) cells to the total number of cells in the EGL did not differ between the GR and control groups at 50 and $60 \mathrm{dg}$. The density of NeuN-IR cells was greater in GR fetuses than in controls at $60 \mathrm{dg}(P<0.05)$ but not at $50 \mathrm{dg}$. At one week after birth, the EGL was just one cell thick, and only a few NeuN-IR cells could be observed in both groups. TUNEL assays performed to enable the evaluation of apoptosis in the cerebellar EGL revealed that cell death was not affected by hypoxia at $50 \mathrm{dg}, 60 \mathrm{dg}$, and one week after birth.

Conclusion: These findings indicate that chronic prenatal hypoxia affects the process of neuronal production late in fetal life, but that this effect does not persist postnatally.

Keywords: Hypoxia, Cerebellum, Guinea pig

\footnotetext{
* Correspondence: jyh1483@chosun.ac.kr

${ }^{2}$ Department of Anatomy, School of Medicine, Chosun University, 375

Seosuk-dong, Dong-Gu, Gwangju 501-759, Korea

Full list of author information is available at the end of the article
} 


\section{Background}

An inadequate intrauterine environment is thought to affect fetal brain development [1,2]. Very low birth weight, which is affected by intrauterine growth restriction (IUGR), is associated with a regional reduction in brain volume [3] and an increased risk of white matter injury [4].

Abnormal brain development is associated with neurological disorders such as cerebral palsy [5], schizophrenia [6], and cognitive [7] and learning/memory [8] deficits. These neurological problems are affected by conditions that cause chronic placental insufficiency (CPI), such as malnutrition, hypoxia, and acidemia [9]. Several models of CPI have been used to determine the relationship between neurological disorders and abnormal brain development. For example, Duncan et al. reported that late gestational CPI affects the morphology and neurotrophin expression of the postnatal sheep brain [10]. Moreover, IUGR induced by CPI in the guinea pig was shown to affect the maturation of myelin [11].

In a recent guinea pig model of CPI, which was induced by uterine artery ligation, the growth of the cellular and neuropil layers were found to be reduced in the cerebellum, without concomitant changes in the expressions of immunoreactivity to either brain-derived neurotrophic factor (BDNF)-IR or tropomyosin receptor kinase B (TrkB)-IR [12]. Some studies have implicated hypoxia in structural alterations of the cerebellum during development. First, significant reductions have been observed in the width of the molecular and inner granular layers [13]. Second, the size and number of Purkinje cells were also found to be reduced [14]. Unlike changes in other layers of the cerebellum, the changes in the cerebellar external granular layer (EGL) induced by chronic prenatal hypoxia are not well understood. Granule cells comprise half of the neurons in the central nervous system, and their precursors are dispersed within the cerebellar EGL [15].

The effects of hypoxia on the development of mature neurons in the EGL were investigated in the present study using a guinea pig CPI model.

\section{Methods}

\section{Animal surgery}

All of the animal experiments were approved by Chosun University Institutional Animal Care and Use Committee (approval number CIACUC2012-A0007). Pregnant DunkinHartley guinea pigs were obtained from a certified breeder (Central Laboratory Animals, Korea). Unilateral uterine artery ligation was performed as reported by Mallard et al. [16]. Briefly, animals were anesthetized with Zoletil (10 mg/kg; Virbac, France) and xylazine (0.15 mg/kg; Bayer, Germany), which were administered via intramuscular injection at 30 to 32 days of gestation (dg) - with term defined as approximately $67 \mathrm{dg}$. After shaving, a midline incision was made below the umbilicus under aseptic conditions. The fat pad of one of the uterine horns was exposed and ligated with silk sutures (4/0). After the procedure, the abdomen was disinfected with a solution of povidone-iodine (Green Medical, Korea). The animals $(n=28)$ were then caged and raised in the same environment.

\section{Tissue preparation}

Fetuses were delivered by cesarean section at $50 \mathrm{dg}$ $(n=14)$ and $60 \mathrm{dg}(n=14)$. Fetuses from the unoperated horn were assigned to the control group (50 dg, $n=7 ; 60$ $\mathrm{dg}, n=7)$, and those from the other, ligated horn were assigned to the growth-restricted (GR) group (50 dg, $n=$ 7 ; $60 \mathrm{dg}, n=7)$. On removal from the uterine horn, fetuses were fixed in $4 \%$ paraformaldehyde (PFA) solution. Fetal cerebella were separated from their brainstem and postfixed in $4 \%$ PFA at $4^{\circ} \mathrm{C}$. After two days, the cerebella were washed with water, dehydrated through a graded ethanol series, and then embedded in paraffin. Serial $12 \mu \mathrm{m}$ thick sections of the cerebellum were mounted on gelatin-coated slides (Fisher Scientific, USA).

\section{Immunohistochemistry}

The sections were deparaffinized, rehydrated, and then washed in 0.1 M PBS ( $\mathrm{pH} 7.4$ ). A microwave antigenretrieval step was performed with $0.01 \mathrm{M}$ sodium citrate buffer ( $\mathrm{pH}$ 6.0). After cooling, the sections were treated with $0.3 \%$ hydrogen peroxide for 20 minutes to block endogenous peroxidase and then rinsed in PBS. They were blocked with normal horse serum in $0.5 \%$ BSA solution for 30 minutes at room temperature and then incubated with one of the following primary antibodies overnight at $4^{\circ} \mathrm{C}$ : rabbit anti-hypoxia-induced factor $1 \alpha$ (Hif1 $\alpha ; 1: 500$, Abcam, UK), rabbit anti-Pax6 (1:1,000, Abcam), goat anti-NeuroD (1:100, Santa Cruz, USA), and mouse anti-NeuN (1:100; Millipore, USA). The next day, the sections were rinsed several times in PBS, and the immunoreactivity visualized using a biotinylated antimouse IgG, the avidin-biotin-peroxidase (ABC) detection system (Vectastain ABC Elite Kit, Vector Laboratories), and the chromogen 3,3'-diamino-benzidine. Thionin counterstaining was performed, after which the sections were dehydrated and coverslipped using PolyMount mounting medium (Polysciences, USA).

\section{TUNEL staining}

Terminal deoxynucleotidyl transferase dUTP nick end labeling (TUNEL) assays were performed with the ApopTag Peroxidase In Situ Apoptosis Detection Kit (S7100, Millipore) according to the manufacturer's protocol. 

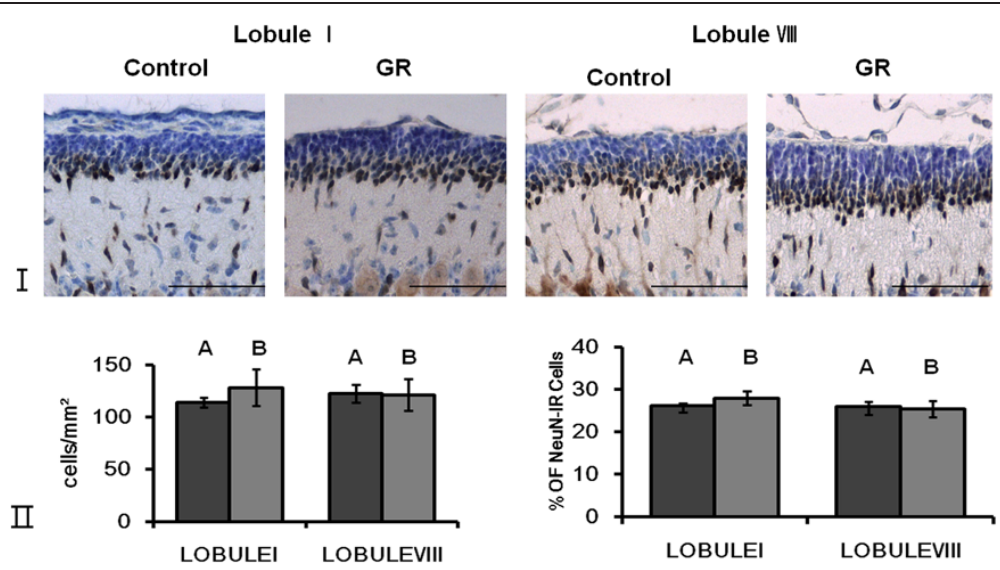

Figure 1 Representative photomicrographs of NeuN-IR cells in the EGL at $\mathbf{5 0} \mathbf{~ d g}$. (I) NeuN-IR cells were located at the bottom of the EGL. Scale bars $=100 \mu \mathrm{m}$. (II) The density and proportion of NeuN-IR cells in the EGL of lobule I and lobule VIII from controls and GR fetuses at $50 \mathrm{dg}$ (A: Control B: GR). The density and proportion of NeuN-IR cells in the EGL did not differ significantly between control and GR fetuses. Values are expressed as a Mean \pm SEM. $(p>0.05)$.

\section{Quantification}

The slides containing stained sections were viewed via a digital CCD camera that was mounted on a light microscope (Olympus BX41, USA). The number of NeuN- and Hif1 $\alpha$-immunoreactive (IR) cells in the EGL of cerebellar lobules I and VIII were counted (immunoreactivity to the other tested antibodies was not observed). The cerebellum was bisected in the midsagittal plane and one-half was embedded in paraffin with the vermal surface as the cutting surface [17]. Sagittal sections of cerebellum that were separated by $300 \mu \mathrm{m}$ were chosen from each animal as described previously [12]. The densities of NeuN-IR cells in the EGL of lobules I and VIII were determined using a counting frame of a defined size placed over five areas selected randomly in each section, and quantified in cells $/ \mathrm{mm}^{2}$. The proportion of NeuN-IR cells was expressed as a percentage of the total number of cells in the EGL.

\section{Statistical analysis}

All data were analyzed using the Statistical Package for Social Sciences (Information Analysis Systems, SPSS, USA). All measurements were compared between the control and GR groups using Student's $t$-test. The level of statistical significance was set at $P<0.05$.

\section{Results}

\section{At 50 dg}

There were no Pax6-IR or NeuroD-IR cells in the EGL at $50 \mathrm{dg}$ (data not shown). NeuN-IR cells were located at the bottom of the EGL (Figure 1). The density and relative proportion (Figure 1) of NeuN-IR cells in the EGL did not differ significantly between control and GR fetuses. TUNEL-positive cells were seen only rarely in the EGL of both groups (Figure 2). The density of Hif $1 \alpha$-IR cells was greater in the GR fetuses than in the controls $(P<0.05$; Figure 3$)$.

\section{At 60 dg}

There were also no Pax6-IR or NeuroD-IR cells in the EGL at $60 \mathrm{dg}$. Furthermore, the pattern of cerebellar NeuN immunostaining in 60-dg fetuses was similar to that in 50-dg fetuses (Figure 4). The density of NeuN-IR cells was greater in GR fetuses than in controls at $60 \mathrm{dg}$ $(P<0.05$; Figure 4$)$. The relative proportion of NeuN-IR cells in the EGL did not differ significantly between the two groups (Figure 4). Few TUNEL-positive cells could

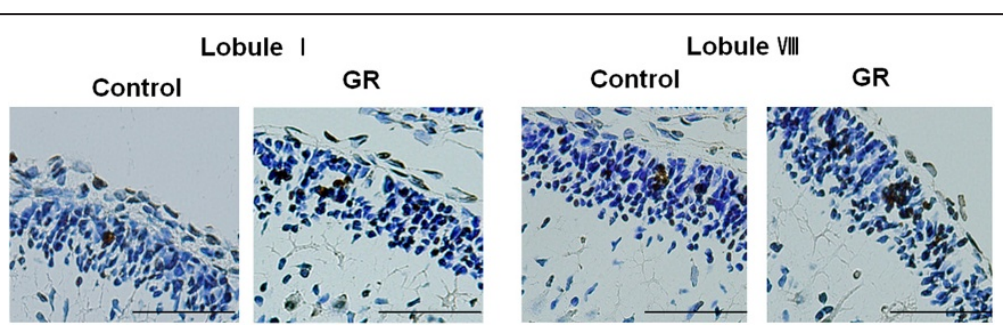

Figure 2 Representative photomicrographs of TUNEL-positive cells in the external granular layer (EGL) at 50 dg. TUNEL-positive cells were seen only rarely in the EGL of both groups. Scale bars $=100 \mu \mathrm{m}$. 


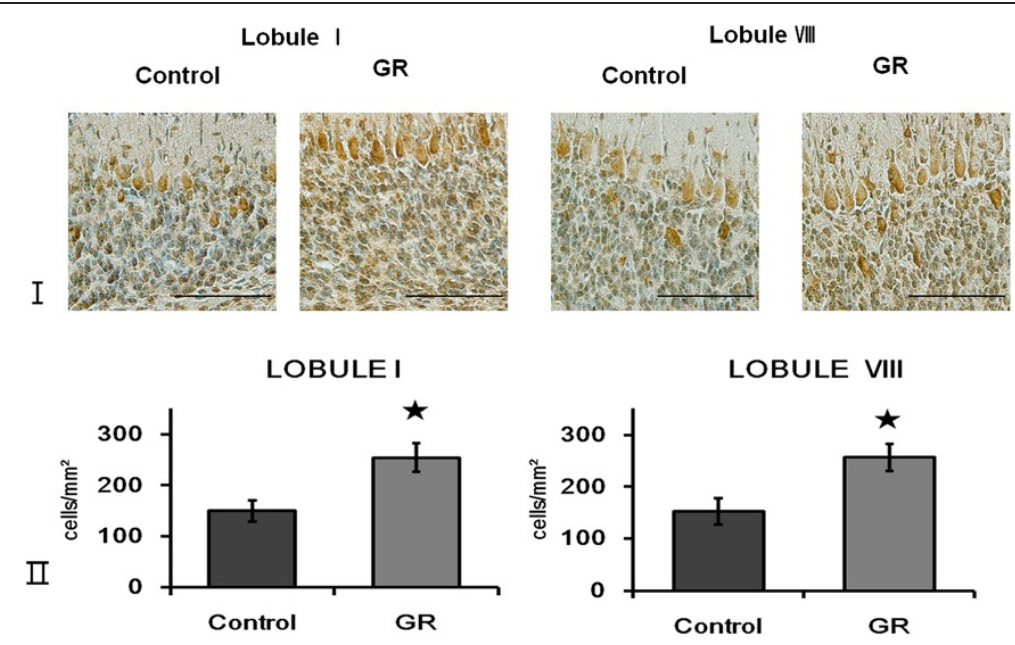

Figure 3 Expression of Hif1a in the IGL at $\mathbf{5 0}$ dg. (I) Hif1 a Immunoreactivity in the IGL of lobule I and VIII from a control and a GR fetus at 50 dg. Scale bars $=100 \mu \mathrm{m}$. (II) The density of Hif1 a-IR cells in the IGL of lobule I and lobule VIII from controls and GR fetuses at $50 \mathrm{dg}$. The density of Hif1a-IR cells was greater in the GR fetuses than in the controls. Values are expressed as a Mean \pm SEM. $\star p<0.05$.

be observed in the EGL of either group (Figure 5). The density of Hif1 $\alpha$-IR cells was greater in GR fetuses than in controls $(P<0.05$; Figure 6$)$, and did not differ significantly from that in $50-$ dg fetuses.

\section{At one week after birth}

At one week after birth, the EGL was forming a one cellthick layer in both of the lobules examined. NeuN-IR cells were only rarely observed in the EGL (data not shown), and Pax6-IR and NeuroD-IR cells could not be found at all. As with NeuN-IR, TUNEL-positive cells were observed only very rarely in the EGL (data not shown).

\section{Discussion}

The guinea pig is a useful model of brain development because it has a relatively long gestation period (with term occurring at approximately $67 \mathrm{dg}$ ) and most of the development occurs in utero. Lobules I and VIII were selected for examination because they are the first and one of the last lobules respectively, to mature [18]. Unilateral uterine artery ligation was performed at $30 \mathrm{dg}$, and we studied fetuses at 50 and $60 \mathrm{dg}$. It has been reported previously that the cerebellum of 30 -dg fetuses appeared immature and consisted of undifferentiated neurons [16]. Furthermore, Jansson et al. [19] reported
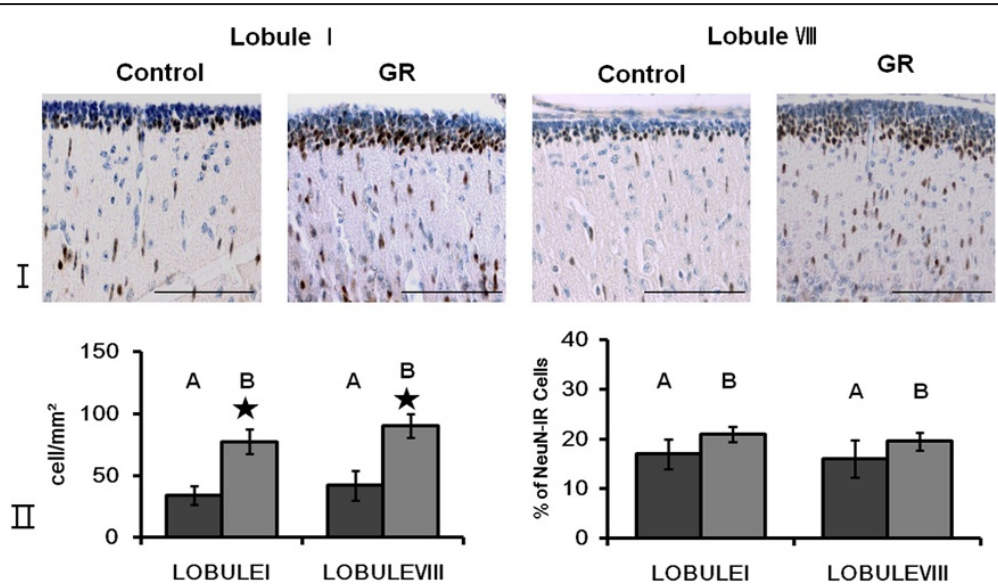

Figure 4 The number of mature neurons in the EGL was greater in GR fetuses than in the controls at 60 dg. (I) Representative photomicrographs of the NeuN immunoreactivity in the EGL of lobule I and VIII from a control and a GR fetus at 60 dg. Scale bars= $100 \mu \mathrm{m}$. (II) The density of NeuN-IR cells in the EGL of lobule I and lobule VIII from controls and GR fetuses at $60 \mathrm{dg}$ (A: Control B: GR). The density of NeuN-IR cells was increased in both lobules of GR fetuses compared to controls at $60 \mathrm{dg}$. However the proportion of NeuN-IR cells did not differ in both lobules of GR fetuses compared to controls at $60 \mathrm{dg}$. Values are expressed as a Mean \pm SEM. $\star p<0.05$. 


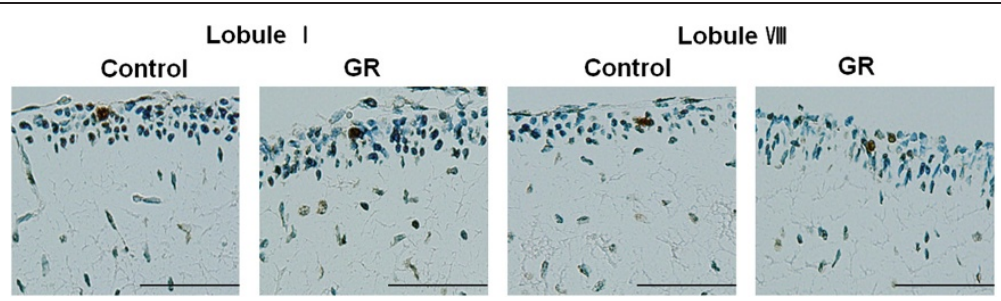

Figure 5 TUNEL-positive cells in the external granular layer (EGL) of lobule I and VIII from a control and a growth-restricted (GR) fetus at 60 dg. Few TUNEL-positive cells could be observed in the EGL of either group. Scale bars $=100 \mu \mathrm{m}$.

that fetal weight and placental blood flow were decreased in guinea pigs at 45,55 , and $65 \mathrm{dg}$. Therefore, we expected that neurogenesis in the guinea pig cerebellum would be reduced at 50 and $60 \mathrm{dg}$. However, the relative proportions of NeuN-IR cells did not differ significantly between control and GR animals at 50 and $60 \mathrm{dg}$, and the pattern of immunoreactivity was similar between 50 and $60 \mathrm{dg}$. On the other hand, the density of NeuN-IR cells was increased in GR fetuses at $60 \mathrm{dg}$.

NeuN is a neuronal-specific nuclear protein [20] and NeuN immunoreactivity is used to obtain independent estimates of total numbers of neuronal and nonneuronal cells [21]. At $50 \mathrm{dg}$, the proportion of NeuN-IR cells relative to the total number of cells and the density of NeuN-IR cells in the EGL did not differ between normal and hypoxic cerebella. This suggests that chronic hypoxia does not have a comprehensive effect on neuronal cell formation at $50 \mathrm{dg}$. However, a significant reduction in the number of NeuN-positive neuronal nuclei was demonstrated in the developing chick brain [22] and Rees et al. [23] reported a reduction in the density of cells undergoing mitosis in the EGL layer of hypoxemic fetal sheep compared to controls. The differences in the findings between the present study and others may be at least partly attributable to the use of different species of experimental model and to the degree of hypoxia imposed. The expression of Hif1 $\alpha$ is induced by hypoxiaischemia [24]. Therefore, while the presence of HIF1 $\alpha$ immunoreactivity in the fetal tissue at $50 \mathrm{dg}$ demonstrates that at that time the fetus had been in a hypoxic

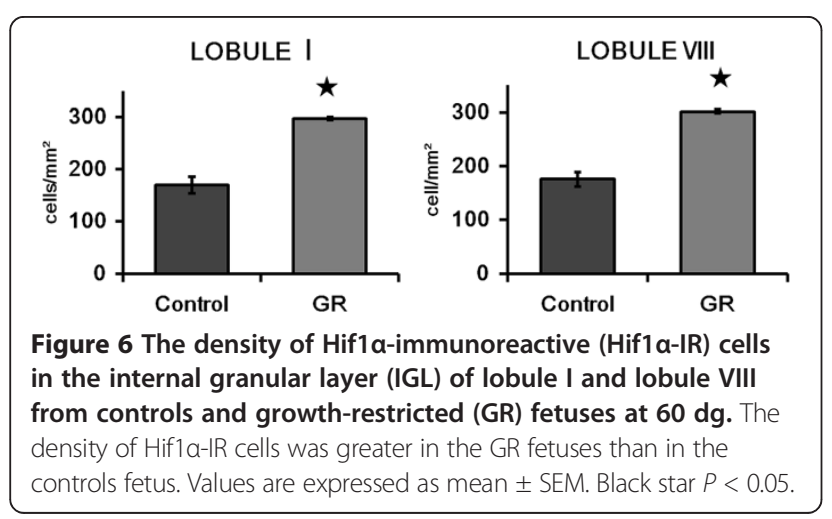

condition, it does not indicate whether that hypoxia was mild or severe. Jansson et al. performed unilateral uterine artery ligation at 30 to $32 \mathrm{dg}$ in the guinea pig [19]. Placenta blood flow was reduced by $25 \%$ at $45 \mathrm{dg}$ and by almost $50 \%$ at $55 \mathrm{dg}$ in the ligated horn [19]. Further experiments are needed to determine the degree of hypoxia that is established using this ligation model, and the degree to which it varies between animals at the same gestation time points. However, since the major developmental events in the guinea pig brain occur before $48 \mathrm{dg}$ [25], it is possible that ligation of the uterine artery affects brain development at $50 \mathrm{dg}$.

At $60 \mathrm{dg}$, the proportion of NeuN-IR cells did not differ between control and GR cerebella. However, the density of NeuN-IR cells was increased in GR fetuses, indicating a larger total cell volume. The density of HIF $1 \alpha$-IR cells did not differ between 60 and $50 \mathrm{dg}$, which implies that the hypoxic condition had not changed between these two time periods. The TUNEL assay findings demonstrate that cell death was not affected by the hypoxia induced in this model. Pax6 is a neuronal stem cell marker [26] and NeuroD is implicated in the differentiation of neurons [27]; we observed no cell immunoreactivity to either of these markers, which suggests that neuronal maturation is already complete at this stage (that is, $60 \mathrm{dg}$ ). We performed immunohistochemistry with Pax6 and NeuroD at $30 \mathrm{dg}$, and observed a few Pax6-IR and NeuroD-IR cells. However, the number of sections was insufficient for providing representative data. An earlier-gestation guinea pig model is required to study the early stages of neuron formation. Since in normal brain development the granule cells in the cerebellar EGL migrate medially and the EGL gradually disappears [28], it is possible that the migration of mature neurons was delayed at this time.

At one week after birth, NeuN-IR cells were only rarely observed in the EGL under both the GR and control conditions. As mentioned above, this is the usual process of cerebellum development. In our study, the observed prenatal alterations to the EGL did not persist postnatally. More studies with animals of different ages are required to establish how chronic prenatal hypoxia affects the mature cerebellum. 


\section{Conclusion}

The findings of this study suggest that chronic prenatal hypoxia affects the process of neuronal production late in fetal life, but that this effect does not persist postnatally. The approach used in this study is useful for extending our understanding of neurogenesis in the EGL, and the findings may be useful for elucidating the brain injury caused by prenatal hypoxia.

\section{Abbreviations \\ CPI: Chronic placental insufficiency; Dg: Days of gestation; EGL: External granular layer; GR: Growth-restricted; IR: Immunoreactive.}

\section{Competing interests}

The authors declare that they have no competing interests.

\section{Authors' contributions}

YYJ designed the study and performed the statistical analyses. KYS, HYL, and EYK participated in the surgical procedures. YHJ carried out the immunohistochemistry assay and analyzed the obtained data. All authors read and approved the final manuscript.

\section{Acknowledgement}

This work was supported by a grant from the Clinical Medicine Research Institute of Chosun University Hospital (2010).

\section{Author details}

'Department of Anesthesiology and Pain medicine, Chosun University Hospital, 375 Seosuk-dong, Dong-Gu, Gwangju 501-759, Korea. '2Department of Anatomy, School of Medicine, Chosun University, 375 Seosuk-dong, DongGu, Gwangju 501-759, Korea. ${ }^{3}$ Department of pediatrics, Chosun University Hospital, 375 Seosuk-dong, Dong-Gu, Gwangju 501-759, Korea.

Received: 3 January 2013 Accepted: 24 June 2013

Published: 3 July 2013

\section{References}

1. Barrett $\mathrm{RD}$, et al: Destruction and reconstruction: hypoxia and the developing brain. Birth Defects Res C Embryo Today 2007, 81(3):163-176.

2. Rees $S$, Harding R: Brain development during fetal life: influences of the intra-uterine environment. Neurosci Lett 2004, 361(1-3):111-114.

3. Bradley SPAWA, et al: Regional brain volumes and their later neurodevelopmental correlate in term and preterm infants. Pediatrics 2003, 111(5):939-948.

4. Skranes J, et al: Clinical findings and white matter abnormalities seen on diffusion tensor imaging in adolescents with very low birth weight. Brain 2007, 130(3):654-666.

5. Liu JJ, Li ZZ, Lin QQ: Intrauterine growth retardation and cerebral palsy. Zhonghua yu fang yi xue za zhi (Chinese journal of preventive medicine) 2001, 35(6):390-393.

6. Mallard ECE, et al: Ventriculomegaly and reduced hippocampal volume following intrauterine growth-restriction: implications for the etiology of schizophrenia. Schizophr Res 1999, 40(1):11-21.

7. Saigal SS, et al: Cognitive abilities and school performance of extremely low birth weight children and matched term control children at age eight years: a regional study. J Pediatr 1991, 118(5):751-760.

8. Dringenberg $\mathrm{HCH}$, et al: Spatial learning in the guinea pig: cued versus non-cued learning, sex differences, and comparison with rats. Behav Brain Res 2001, 124(1):97-101.

9. Neerhof MG, Thaete LG: The fetal response to chronic placental insufficiency. Semin Perinatol 2008, 32(3):201-205.

10. Duncan JR, et al: Neurotrophin expression in the hippocampus and cerebellum is affected by chronic placental insufficiency in the late gestational ovine fetus. Dev Brain Res 2004, 153(2):243-250.

11. Tolcos $M$, et al: Intrauterine growth restriction affects the maturation of myelin. Exp Neurol 2011, 232(1):53-65.

12. Dieni SS, Rees SS: BDNF and TrkB protein expression is altered in the fetal hippocampus but not cerebellum after chronic prenatal compromise. Exp Neurol 2005, 192(2):265-273.
13. Rees $\mathrm{S}$, et al: Hypoxemia near mid-gestation has long-term effects on fetal brain development. J Neuropathol Exp Neurol 1999, 58(9):932-945.

14. Hausmann R, Seidl S, Betz P: Hypoxic changes in Purkinje cells of the human cerebellum. Int J Legal Med 2007, 121(3):175-183.

15. Altman JJ, Bayer SAS: Embryonic development of the rat cerebellum. II. Translocation and regional distribution of the deep neurons. J Comp Neurol 1985, 231(1):27-41.

16. Mallard CC, et al: Reduced number of neurons in the hippocampus and the cerebellum in the postnatal guinea pig following intrauterine growth-restriction. Neuroscience 2000, 100(2):327-333.

17. Nathaniel EJ, et al: Effect of exogenous thyroxine on the development of the Purkinje cell in fetal alcohol effects in the rat. Exp Mol Pathol 1999, 67(3):175-191.

18. Altman Jj: Autoradiographic and histological studies of postnatal neurogenesis. 3. Dating the time of production and onset of differentiation of cerebellar microneurons in rats. J Comp Neurol 1969, 136(3):269-293.

19. Jansson TT, Thordstein MM, Kjellmer II: Placental blood flow and fetal weight following uterine artery ligation. Temporal aspects of intrauterine growth retardation in the guinea pig. Biol Neonate 1986, 49(3):172-180.

20. Mullen RJR, Buck CRC, Smith AMA: NeuN, a neuronal specific nuclear protein in vertebrates. Development 1992, 116(1):201-211.

21. Herculano-Houzel S, Lent R: Isotropic fractionator: a simple, rapid method for the quantification of total cell and neuron numbers in the brain. J Neurosci 2005, 25(10):2518-2521

22. Rodricks $\mathrm{CL}$, et al: The effect of hypoxia on the functional and structural development of the chick brain. Int J Dev Neurosci 2010, 28(4):343-350.

23. Rees SS, et al: The vulnerability of the fetal sheep brain to hypoxemia at mid-gestation. Brain research. Dev Brain Res 1997, 103(2):103-118.

24. Bergeron M, et al: Induction of hypoxia-inducible factor-1 (HIF-1) and its target genes following focal ischemia in rat brain. Eur J Neurosci 1999, 11(12):4159-4170.

25. Dobbing JJ, Sands JJ: Growth and development of the brain and spinal cord of the guinea pig. Brain Res 1970, 17(1):115-123.

26. Wang $L$, et al: Basal progenitor cells in the embryonic mouse thalamus - their molecular characterization and the role of neurogenins and Pax6. Neural Dev 2011, 6(1):35-35.

27. Miyata TT, Maeda TT, Lee JEJ: NeuroD is required for differentiation of the granule cells in the cerebellum and hippocampus. Genes Dev 1999, 13(13):1647-1652.

28. Altman JJ, Bayer SAS: Prenatal development of the cerebellar system in the rat. II. Cytogenesis and histogenesis of the inferior olive, pontine gray, and the precerebellar reticular nuclei. J Comp Neurol 1978, 179(1):49-75.

doi:10.1186/1866-1955-5-17

Cite this article as: So et al:: The effect of chronic prenatal hypoxia on the development of mature neurons in the cerebellum. Journal of Neurodevelopmental Disorders 2013 5:17.

\section{Submit your next manuscript to BioMed Central and take full advantage of:}

- Convenient online submission

- Thorough peer review

- No space constraints or color figure charges

- Immediate publication on acceptance

- Inclusion in PubMed, CAS, Scopus and Google Scholar

- Research which is freely available for redistribution 